\title{
Effects of colour and chromatic light on depth perception
}

\author{
Deniz Atlıa ${ }^{a}$ Nilgün Olguntürk ${ }^{b}$, Rengin Aslanoğlu $\mathbb{C}^{b}{ }^{b}$, Dragan Sekulovski ${ }^{c}$ and Pieter Seuntiens ${ }^{c}$ \\ ${ }^{a}$ Theater Department, Faculty of Music and Performance Arts, Bilkent University, Ankara, Turkey; ${ }^{b}$ Department of Interior Architecture and \\ Environmental Design, Faculty of Art, Design, and Architecture, Bilkent University, Ankara, Turkey; ' Signify Research, Eindhoven, The \\ Netherlands
}

\begin{abstract}
Space perception is the ability to estimate the three-dimensional layout of our environment from the arrangement of individual objects, their location and size. While estimating every distance in the three-dimensional environment, the human visual system uses a number of physical cues and depth perception can be affected by a variety of factors; reflectance, colour, and texture. The main purpose of this study is to understand the effect of chromaticity combinations on depth perception. A forced-choice paired comparison test was used to evaluate distance differences between colour combinations created by chromatic light in background and coloured objects in front. The experiments indicated perceptual variations in-depth assessments between different participants, which needed to be taken into consideration. The findings suggest a significant effect of some colour combinations on depth perception.
\end{abstract}

\section{ARTICLE HISTORY}

Received 18 October 2019

Accepted 2 December 2019

\section{KEYWORDS}

Chromatic light; colour combinations;

three-dimensional layout; depth assessments

\section{Introduction}

Understanding the interaction between surface colours and illumination chromaticity enables effective use of space in the built environment; especially in interior architecture, lighting and stage design. As all human with normal visual abilities identifies their environments as three-dimensional layouts, they always estimate it with free space perception; there are two potential sources of information that affect it: luminance contrast and colour contrast (1). Space perception, in other words, depth perception, is the ability to estimate the three-dimensional layout of our environment with both eyes (stereopsis) from the arrangement of individual objects, their location and size. In that context, many studies focus on the perception of distance as the central problem of space perception. In this distance estimation, the human visual system uses a number of physical attributes, or cues, from the environment $(2,3)$.

Among the monocular cues, colour is one of the most debated. Starting with the study of Luckiesh (4) done with an apparatus of red and blue letters that can be moved back and forth, colour has long been studied as an influencing factor for judgment of distance. The effect of brightness and saturation on depth perception, both absolute and with relation to the background has been studied extensively. According to the studies, the objects or the images with higher brightness and/or saturation were perceived closer to the participants $(5,6)$. Other than that, the effect of brightness contrast on the judgment of distance was also studied by Ichihara, Kitagawa and Akutsu (7), and Dresp and Guibal (8). These studies include a distance comparison of coloured objects when they were presented in front of coloured backgrounds. In these studies, the coloured patch which resembled the background the most was perceived more distant than the other coloured patches. All the above studies were done with coloured objects or patches under achromatic light however, there are only a few studies which explore the chromatic light effect on depth perception. In one of the earlier studies, an experiment was designed with red neon or blue neon and argon lights (9). The results showed blue light was perceived nearer even when it was at a larger distance than red light. However, the results of Pillsbury and Schaefer's (9) study is insufficient for understanding the dominant attribute of the light and/ or colour affecting the perception. Similarly, in a study which used chromatic light and coloured surfaces, Huang (10) used different intensities and different hues in his experiment which masks and confuses the causes of the effect he searched for. The study presented in this

CONTACT Rengin Aslanoğlu rengin.kocaoglu@bilkent.edu.tr $\Theta$ Department of Interior Architecture and Environmental Design, Faculty of Art, Design, and Architecture, Bilkent University, Ankara 06800, Turkey 
paper attempts to bridge this gap by studying the effect of chromatic light and chromaticity combinations on depth perception.

It is known that colour of objects has a considerable effect on depth perception in the visual field. Besides colour, light is also another concern in depth perception, which makes colour visible in the physical environment. Thus, colour as an independent cue for distance perception has two different conditions to be applied. First one is the coloured surface and second aspect is the colour of the light. While colour was taken as an independent cue for distance judgment, its parameters of hue, saturation and brightness should also be considered. In the outdoor study of Mount, Case, Sanderson and Brenner (6), they mentioned about a hue effect on perceived distance. In the experiment, they compared equal brightness of coloured and gray papers under sunlight. As a result, they expressed that each colour is judged nearer than its equal brightness gray and the hues appear closer when viewed against the dark rather than light colour. They also pointed out that, besides the differences observed in distance perception because of hue, saturation and brightness contrast are also effective in perceiving the distance of coloured surfaces. They (6) mentioned about advancement in the apparent position of colour when the saturation of its colour is increased from its background. Similarly, according to Egusa's (11) findings, there were also differences in hues in terms of perceived depth. He (11) confirmed that green and blue difference is smaller than the red and green one in which the red one appears nearer. $\mathrm{He}$ (11) also noted that if the higher saturated colour is red or green, they are judged nearer, but such an effect cannot be said to blue. In the study of Camgöz, Yener and Güvenç (12) on every background colour viewed through a computer monitor, the coloured squares having maximum saturation and maximum brightness were found to attract the most attention, thus appearing to be nearer. As another addition to the saturation effect, Bailey, Grimm and Davoli (5) studied with less saturated colours than the previous works. In the experiment with different coloured teapots and backgrounds on the computer screen, they obtained similar results with previous works as observing the brighter colours appearing closer than the dark ones. In spite of differences in hue and saturation, brightness effects in distance perception were obtained most dominantly. Johns and Sumner (13) studied with an apparatus in which differently coloured papers were set. Participants were asked to equalize the distances of these coloured papers. As a result, they stated that at a constant distance, light colours appeared nearer than dark colours. Michel (2) mentioned the effect of brightness on perceived distance as gamma movement. As the object brightness is increased, the object 'appears to advance toward the viewer from its initial fixation point' $(2$, p. 12) and it returns its former position when the brightness is decreased.

This study seeks to find the effect of colour and coloured light on peoples' perception of distance by using a forced-choice paired comparison test for exploring the distance differences between colour combinations created by chromatic light in the background and coloured objects in front. According to the results, the effects of chromaticity on depth perception showed variations between participants and different background combinations found to have an effect on depth perception.

\section{The experiment}

Twenty-one students aged between 22 and 29 years participated in the experiment taking place in a controlled 1/1 interior space in Shoplab of Holst Center, at High Tech Campus, Eindhoven. The stimuli consisted of a two-part background which was lit with separately controllable wall washers (is a uniform lighting design system for illuminating of large and vertical surfaces) and two objects in front. Six different colours were used for the background: orange, blue, red, green, warm white $(3000 \mathrm{~K})$ and cool white $(6500 \mathrm{~K})$ (See Table 1$)$. The objects were $45 \mathrm{~cm} \times 45 \mathrm{~cm}$ squares painted in one of the colours orange, blue and gray. The objects were lit with halogen spots $(3000 \mathrm{~K}$ ) (is an incandescent lamp filled with halogen gas) from the top. The brightness and saturation of the lights and objects were matched with each other as much as possible in order to investigate out the hue effect on depth perception.

Each experiment included two sets of colour combinations in which the colour of the objects was equal but the background colours were different. In one of the sets, the background colour was always fixed to cool white light for both sides as a neutral background chromaticity and in the second set background with two different colours adjacent to each other was presented (See Figure 1).

Five of the background colours were paired with cool white light for the second set of combination in each session. Additional to these five colour pairs, the pairs of red-green background and orange-blue background were also presented as they had been found useful in exploring the chromaticity effect on depth perception in the pilot study. For the blue and orange objects, the identical background colour was not displayed. There were 17 object and background colour combinations in total. The right object of the apparatus was fixed and the left one was moved to seven positions determined previously. These seven points were 10,20 and $30 \mathrm{~cm}$ in the front and back of the right object and both objects being in 
Table 1. The CIE L* ${ }^{*}{ }^{*} H^{*}$ values of six background colours (colours were measured with Photo Research Spectra Duo PR 680 and Macro Spectar MS-75 lens).

\begin{tabular}{lccccccc}
\hline $\begin{array}{l}\text { Colour of the } \\
\text { background }\end{array}$ & Lightness $(\mathrm{L})$ & Chroma $(\mathrm{C})$ & Hue $(\mathrm{H})$ & $\begin{array}{c}\text { Colour of } \\
\text { Background }\end{array}$ & Lightness (L) & Chroma (C) & Hue (H) \\
\hline Warm white & 58.69 & 3.7 & 0 & Red & 59.54 & 54.79 & 10.13 \\
Cool white & 58.37 & 77.92 & 274 & Blue & 55.64 & 55.98 & 244.39 \\
Orange & 57.89 & 56.89 & 48.13 & Green & 58.68 & 54.76 & 168.34 \\
\hline
\end{tabular}
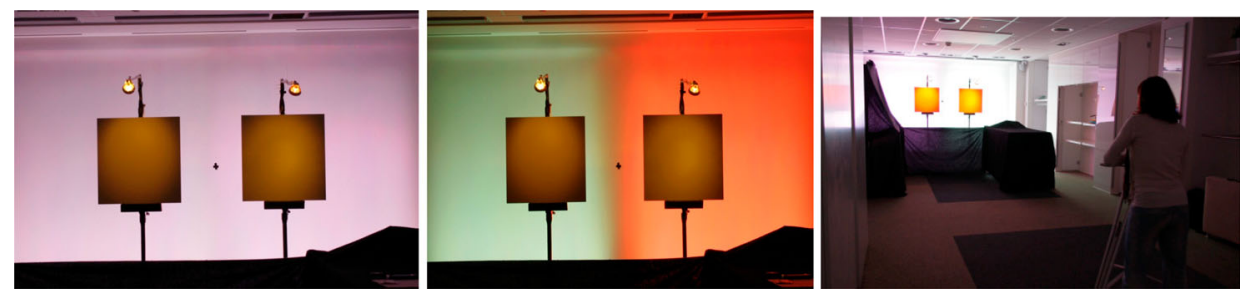

Figure 1. Example stimuli demonstrating background chromaticity variations. Left: baseline stimulus, middle: colour combination stimulus, right: during the experiment.

the same distance. Each point was presented 10 times randomly during the experiment, resulting in 70 points for each background. The stimulus was seen from $10 \mathrm{~m}$ viewing distance (in order to avoid the undesired effects of the depth cues other than colour and minimize the detection of size changes as the stimulus changed) with a chinrest by the participant in order to stabilize head movements. Also, a black cross between the two objects was arranged for participants to focus their eye on to concentrate directly onto the colours but not to the size or shape of the objects. The participants were informed before the experiment about where to focus and why. To minimize measurement error, a forced-choice paired comparison was used. The participants were asked which one of the objects appeared closer to them, left or the right one. The experiment was conducted monocularly to avoid the effects of binocular cues and to make sure that the obtained distance differences were derived because of only. The participants took the dominant eye test and they took the experiment with their dominant eye, they judged the stimuli using monocular viewing at a distance of $10 \mathrm{~m}$.

\section{Results and discussion}

As Michel (2) stated, for defining the perception of distance in space perception 'knowledge of the depth cues' such as colour, relative size, relative location, superposition, and occlusion, linear and aerial perspectives should be taken into consideration. Accordingly, in the field of depth perception, colour is one of the most discussed cues which has critical works on it (14), however it has been analysed with small coloured chips and mock-up designs $(5-7,10)$. Besides, all of them have generally examined brightness and saturation attributes of colour and their influence on depth perception. There are no studies exploring hue effect on depth perception in $1 / 1$ scaled interior spaces with the combination of object colour and light colour. The results of this research are important to fill the gap in the literature about chromaticity effects in interior spaces. Thus, this study explored the effects of different colours and different colour combinations on depth perception in interior spaces. It was hypothesized that there are different depth perceptions according to the background hue and its combination with objects which has different hues. Another hypothesis was that there are differences in-depth perception depending on the colour temperature of background lit and object hue in front of it. The differences in the perception were analysed depending on object colour and background colour.

The responses of the repeated measurements were aggregated per participant to compute an estimate of the probability of an object being observed in front of another object, given their colour, the background colours and the relative distance between the objects. Based on the computed probabilities, a psychometric curve was fitted. To compute the distance at which the objects were perceived equidistant the $50 \%$ points on the psychometric curves were used. The standard error of the result was estimated using a parametric bootstrap procedure.

Results showed a surprising, but consistent interobserver variance in-depth perception in the right and left visual field. A repeatable, participant dependent offset of up to $25 \mathrm{~cm}$ for two neutral chromaticity objects on a neutral background was demonstrated. For some observers, the object in the right visual field appeared 

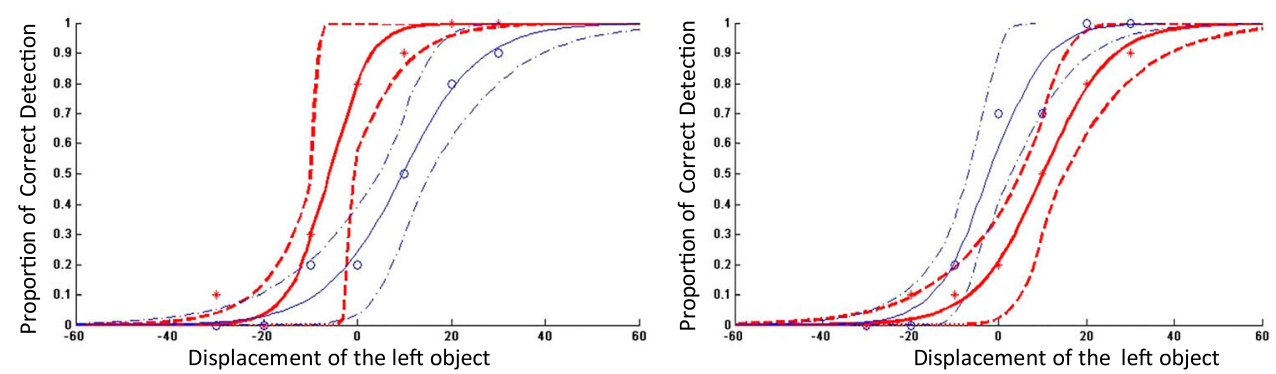

Figure 2. Example psychometric curves for a warm white-cool white background pair. The blue line represents the baseline results. Left: orange objects, $-15 \mathrm{~cm}$, right: blue object, $12 \mathrm{~cm}$.

Table 2. Summary of the results.

\begin{tabular}{llll}
\hline Background pair & Orange object & Blue object & Gray object \\
\hline Cool white-blue & Cool white & N/A & Blue \\
Cool white-green & no sig. difference & no sig. difference & Green \\
Cool white-red & Red & Red & Red \\
Cool white-orange & N/A & no sig. difference & Orange \\
Warm white-cool white & Warm white & Cool white & no sig. difference \\
Red-green & Red & Green & Green \\
Orange-blue & N/A & N/A & Blue \\
\hline
\end{tabular}

closer, and for some in the left. To correct for this effect, the baseline difference was subtracted from all the colour combination results. After correcting for the baseline differences, the corrected distance was used as a dependent variable in an ANOVA procedure. The background colours and the colour of the objects were used as independent variables. A significant effect of both colour and colour combination on depth perception was found, $\mathrm{F}$ $(2,34)=13.83, p<0.001$. Hochberg's GT2 test revealed that there were different depth perceptions occurring between orange and the other two object colours (blue and gray). Cooler objects were generally found to be perceived as further away than warmer ones, as were the achromatic objects.

When the colour combinations were analysed the effect was mostly increased by increasing the chromaticity difference with the background. As expected there were no significant effects of colour temperature when warm white and cool white background pair was displayed with gray objects. The orange object had a tendency to be perceived nearer in front of cool white background and the blue object had a tendency to be perceived nearer in front of warm white background (See Figure 2). The horizontal axis in the figure represents the displacement of the left object. A negative value denotes a situation where the left object is closer to the observer.

The largest distance difference was obtained with a blue object and cool white-red background combination and similarly, with an orange object and cool whiteblue background combination. Additionally, opponent colours showed similar results. Cool white-red and cool white-green background pairs showed a similar amount of difference in the same direction when displayed with gray objects. The same holds for the cool white-orange and cool white-blue background pairs. No difference was perceived with red-green background pair (See Table 2).

The effect of the hue on the perception of depth was smaller than the inter-observer variance in the baseline, which shows limited effectiveness for chromaticity as a tool for depth perception manipulation. However having limited effectiveness, using the lighting chromaticity, the atmosphere of an environment can be manipulated without changing the overall space perception. Even more, the methodology can be developed further for future psychophysical studies where having a large baseline variation between observers is important.

The results showed some similarities and differences with the literature. Chen, Shi, Tai \& Yun (15) contributed the stereoscopic depth perception field by examining the changes in depth perception caused by hue variations. Their results showed that disparity range varies with hue;

the range for yellow hue is greater than red hue, the latter being greater than blue hue and the disparity range for green hue is smallest. It is well known that a greater disparity range indicates more efficient stereoscopic perception. The conclusion is that the perceived depth is not the same for different hue for a given size of disparity. (15 p. 6)

Thus, their results supports the current study's findings; chromatic features can contribute to depth perception and stereoscopic depth perception can vary with the chromaticity. As Jiménez, Rubino, Hita \& del Barco (16) exposed; the variations in the stereograms along redgreen confusion lines contribute to stereopsis. A similar study by Jiménez, del Barco, Dı'az, Hita \& Romero (17) indicated that red- green variations show a higher 
chromatic contrast than yellow-blue variations. When object colours were analysed, blue and gray coloured objects were found to have similar tendencies in differences, where the orange object generally showed opposite results, which verifies the hypothesis of the study. Blue and gray colours are cooler than the orange object and they were generally perceived farther than the orange one, which shows a similarity to the study carried out by Bailey et. al. (5). According to their (5) study, warmer coloured objects appeared closer than cooler ones. Besides that, mainly there were bigger differences observed with the gray object than the blue or orange object. Mount et. al. (6) mentioned that 'each colour was seen in front of its nearest matching gray' (p. 210). In this study, it is revealed that blue and orange were generally perceived nearer than gray, which had the nearest brightness matching with the other two colours. Another similarity with the literature can be mentioned about red and blue combinations. According to the study of Pillsbury and Schaffer (9), due to chromatic aberration 'red and blue showed especially striking differences' (p. 126). In this study, when blue object sets were analysed, the biggest difference was obtained with cool white-red background pair. Besides, for the orange object, the biggest difference was also perceived with cool white-blue background pair.

The reason for these above-mentioned findings can be explained with the studies which explored the effects of adjacent colours on depth perception. Katz described these different approaches of colours on distance perception as insistency of a colour (18). It is the power of the colour to 'catch the eye and hold it steadily' (14, p. 135). The insistent colour has the proper to appear nearer. The brightness effect also appears as contrast differences in adjacent colours. Payne (18) mentioned that if a colour differs from the background more, it stands farther away from the background. Thus, one of the two colours which are most likely to background would appear more distant than the other. In a similar way, Ichihara, Kitagawa and Akutsu (7) claimed the contrast as an important cue for perceiving the depth of an object. They defined 'area contrast as the difference between the average luminance of the surface area of an object and the average luminance of the background' (7). When area contrast is low, the object looks far from the observer; similarly it looks near when the contrast is high. Michel (2) expressed that for appearance of the surface colours, illumination has great importance because light makes the colours and surfaces visible. As Jimenez, Rubino, Diaz \& del Barco Jiménez's (19) results indicated, the luminance signal contributes to stereopsis as equally as chromatic signals do. Also, Dresp and Guibal (8) found in their study on red colour that 'colour is not an independent depth cue but is strongly influenced by luminance contrast and stimulus geometry' (p. 1). According to Dresp and Guibal (8) the probability of 'near' increases with the luminance contrast. They also mention that if the colours are lighter than the background, the long-wavelength colours like orange are seen in front of the short-wavelength colours like blue. As similar, if the colours are darker than the background, blue is seen in front of the orange.

According to the warm and cool colours theory, there is a fundamental phenomenon about red-green and yellow-blue pairs in terms of colour receptors in the eye (20). The colour receptors are responsible for different wavelengths of colours (S, M, L) and for colour pairs opponent signals processed together; for red-green $\mathrm{L}-(\mathrm{M}+\mathrm{S})$ channels are processed and yellow-blue $\mathrm{S}$ $(\mathrm{L}+\mathrm{M})$ channels are processed $(20)$. In this context, for evaluating all of the background pairs, the object in front of the coloured backgrounds was perceived nearer than it was in cool white background. Furthermore, when the red-green background pair was evaluated, it, however, changed according to the participant, so no big differences were obtained. Besides, when orange-blue background pair was evaluated there was a significant difference. In that case, it is possible to say that different background combinations also have an effect on depth perception. Additionally, for background colour combinations, there was no effect of colour temperature on depth perception when it was judged with gray object; however, depending on object colour, it showed differences in different directions. In order to understand and have detailed information about the reasons for those aforementioned results, further experiments need to be done.

\section{Conclusion}

This study aimed to understand the effect of chromaticity combinations on depth perception. A forced-choice paired comparison test was used to evaluate distance differences between colour combinations created by chromatic light in the background and coloured objects in front. The results of the statistical study showed significant effects of colour and coloured light on depth perception in terms of different baselines and distance perception of participants.

As a limitation of the study, due to the baseline and estimation of the distance variations between participants, a method that had specific measuring points and repetitions were applied. Therefore, each participant took a longer experimental period than expected. Some of the participants could not be taken into consideration for statistical analysis because of the variations between them was conflicting. Consequently, to explore the effects of 
colour on depth perception, the results obtained from the psychometric curves of participants were also interpreted one by one. For further studies, besides examining the effects of colour and chromatic light on depth perception, the details of the visual system and neuroscience should be discussed more since those fields' effects on depth perception are not completely revealed (21). In future studies, to standardize the baseline factor and to provide the perceived distance difference, a starting point for the previously specified 7 points can be determined according to the baselines of each participant instead of keeping it stable for each participant. In other words, if a participant has a deviation of $10 \mathrm{~cm}$, the starting point can be taken as $10 \mathrm{~cm}$ instead of $0 \mathrm{~cm}$ and the distances can be specified as 20, 30 and 40 instead of 10, 20 and 30. In future studies, if binocular viewing makes any difference in-depth perception of colours and colour lights in order to find out more practical usages and applications can be investigated. Besides, how colours and colour combinations affect the room size can be looked into by making a contribution to this study in order to understand if the results obtained from depth perception tests are mostly influenced by the background or object colours. More detailed research can be done in order to understand the reasons why the perception of participants showed so much variation with each other in the study.

\section{Disclosure statement}

No potential conflict of interest was reported by the authors.

\section{ORCID}

Rengin Aslanoğlu (D) http://orcid.org/0000-0002-8002-5069

\section{References}

(1) Kingdom, F.A.; Simmons, D.R. Stereoacuity and Colour Contrast. Vision. Res. 1996, 36 (9), 1311-1319.

(2) Michel, L. Light: The Shape of Space- Designing with Space and Light; Van Nostrand Reinhold: New York, 1996.

(3) Sekuler, R.; Blake, R. Perception, 3rd ed.; Mc Graw- Hill: New York, 1994.

(4) Luckiesh, M. On 'Retiring' and 'Advancing' Colors. Am. J. Psychol. 1918, 29, 182.
(5) Bailey, R.; Grimm, C.; Davoli, C. The Real Effect of Warm-Cool Colors. International Conference on Computer Graphics and Interactive Techniques, Boston, MA, 2006.

(6) Mount, G.E.; Case, H.W.; Sanderson, J.W.; Brenner, R. Distance Judgment of Colored Objects. J. Gen. Psychol. 1956, 55, 207-214.

(7) Ichihara, S.; Kitagawa, N.; Akutsu, H. Contrast and Depth Perception: Effects of Texture Contrast and Area Contrast. Perception 2007, 36, 686-695.

(8) Dresp, B.; Guibal, C.R. Interaction of Color and Geometric Cues in Depth Perception: When does 'Red' Mean 'Near'? Psychol. Res. 2004, 69, 30-40.

(9) Pillsbury, W.B.; Schaefer, B.R. A Note on AdvancingRetreating Colors. Am. J. Psychol. 1937, 49, 126-130.

(10) Huang, K.C. Effects of Colored Lights, Spacing Between Stimuli, and Viewing Distance on Error in a DepthMatching Task. Percept. Mot. Skills 2009, 108, 636-642.

(11) Egusa, H. Effects of Brightness, hue and Saturation on Perceived Depth between Adjacent Regions in the Visual Field. Perception 1983, 12, 167-175.

(12) Camgöz, N.; Yener, C.; Güvenç, D. Effects of Hue, Saturation, and Brightness: Part2: Attention. Color. Res. Appl. 2004, 29, 20-28.

(13) Johns, E.H.; Sumner, F.C. Relation of the Brightness Differences of Colors to their Apparent Distances. J. Psychol. 1948, 26, 25-29.

(14) Sundet, J.M. Effects of Colour on Perceived Depth: Review of Experiments and Evaluation of Theories. Scand. J. Psychol. 1978, 19, 133-143.

(15) Chen, Z.; Shi, J.; Tai, Y.; Yun, L. Stereoscopic Depth Perception Varies with Hues. Opt. Eng. 2012, 51 (9), 097401.

(16) Jiménez, J.R.; Rubino, M.; Hita, E.; del Barco, L.J. Influence of the Luminance and Opponent Chromatic Channels on Stereopsis with Randomdot Stereograms. Vision. Res. 1997, 37 (5), 591-596.

(17) Jiménez, J.R.; del Barco, L.J.; D1'az, J.A.; Hita, E.; Romero, J. Assessment of the Visual Effectiveness of Chromatic Signals for CRT Colour Monitor Stimuli. Displays 2000, 21 (4), 151-154.

(18) Payne, M.C., Jr. Color as an Independent Variable in Perceptual Research. Psychol. Bull. 1964, 61 (3), 199-208.

(19) Jimenez, J.R.; Rubino, M.; Diaz, J.A.; del Barco Jiménez, L. Influence of the Luminance Signal and Red-green and Yellow-blue Opponent Chromatic Signals in Figuralstimuli Stereograms. Optom. Vis. Sci. 1995, 72 (9), 649-655.

(20) Fairchild, M.D. Color Appearance Models, John Wiley \& Sons: New York, 2013.

(21) Chakravarthy, V.S. Pathways of Light. In Demystifying the Brain, Springer: Singapore, 2019; pp 169-210. Ed. 